تأثير جههار رقم جو به صورت دانهُ كامل يا آسياب شده

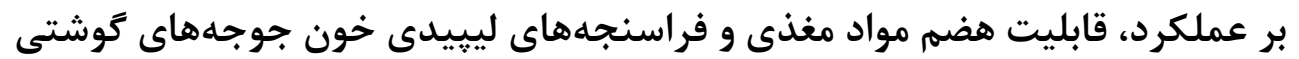

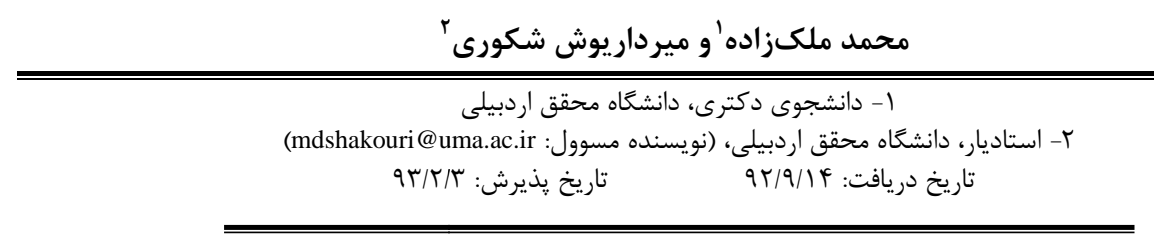

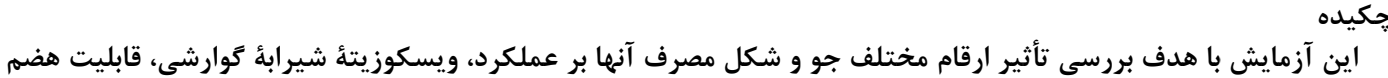

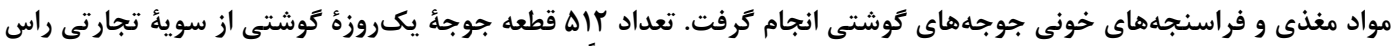

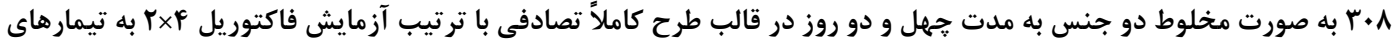

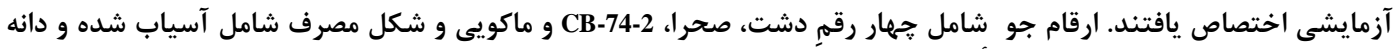

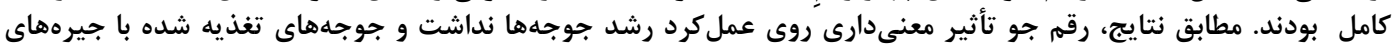

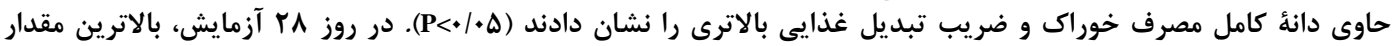

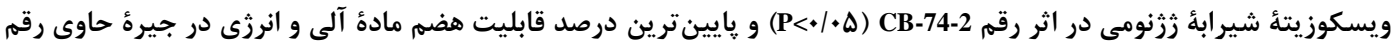

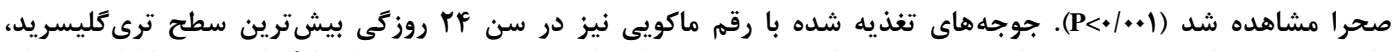

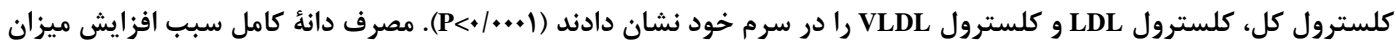

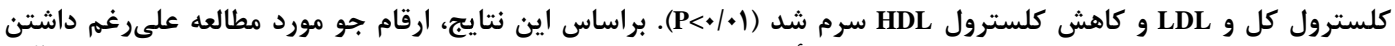

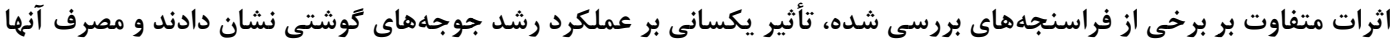

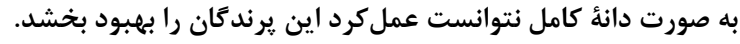

وازههاى كليدى: جوجةُ كَوشتى، دانهُ كامل، رقم جو، عملكرد، قابليت هضم مواد مغذى، ويسكوزيتهُ شيرابهُ زرنومى

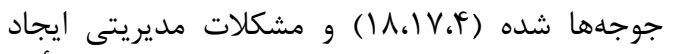

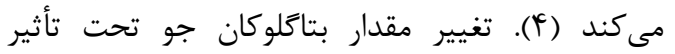

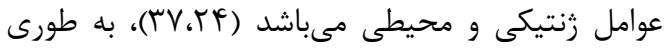

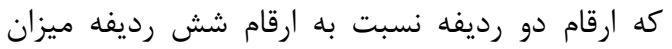

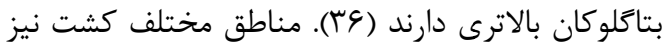

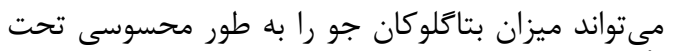

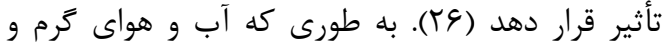

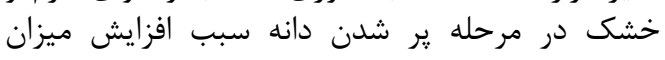

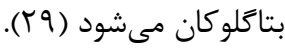

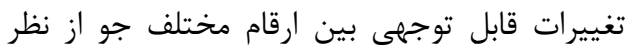

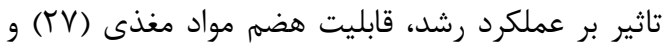

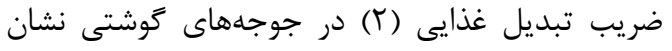
داده شده است.

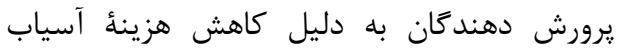

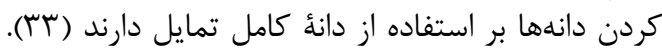

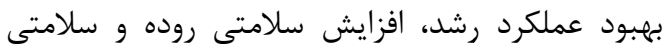

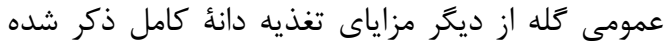

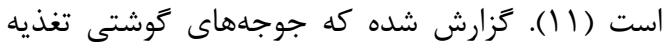

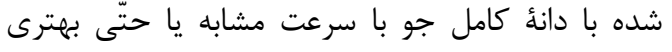

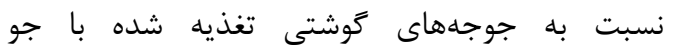
غلطكزده و يا جو آسياب شده رشد مى كنند (بساب).

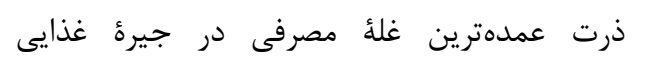

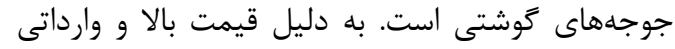

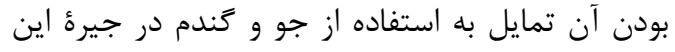

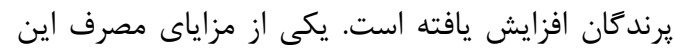

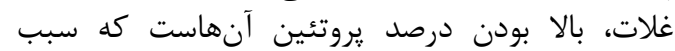

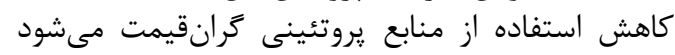

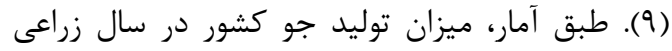

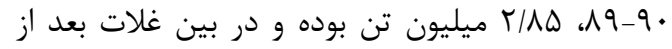

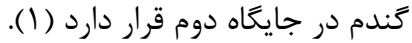

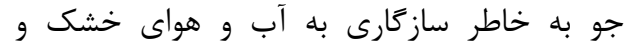

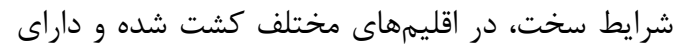

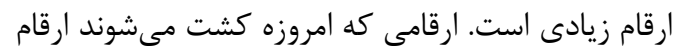

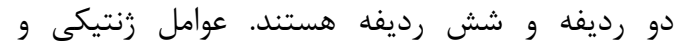

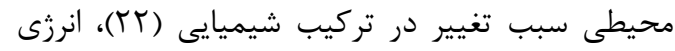

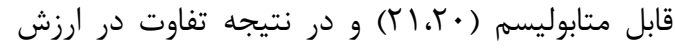
تغذيهاى ارقام مختلف جو مى شود.

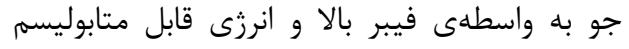

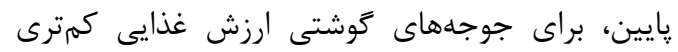

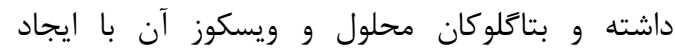

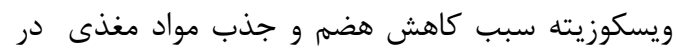




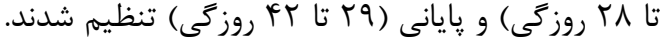

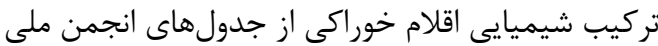

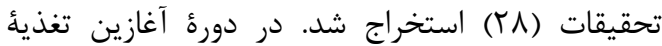

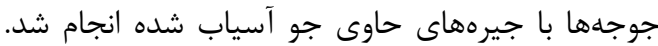

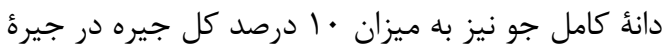

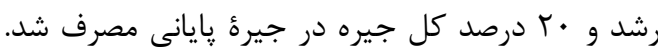

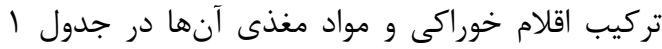
ارائه شده است

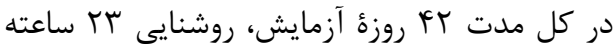

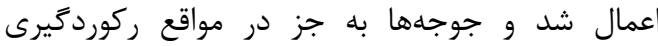

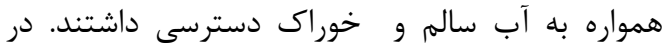

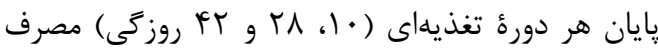

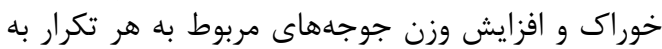

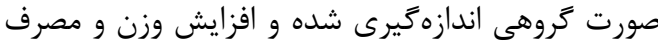

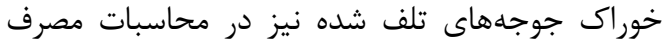

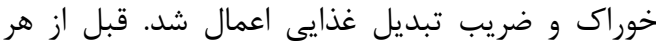

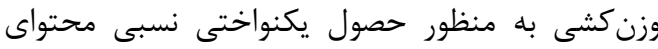

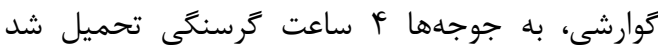
توسط ارك (IV)

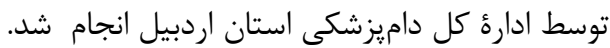

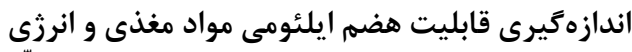

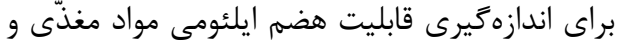

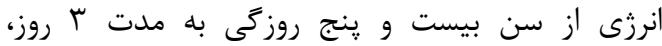

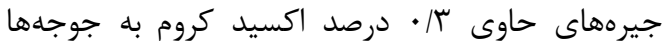

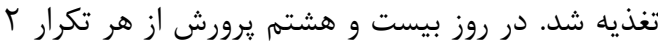

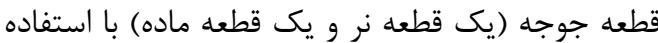

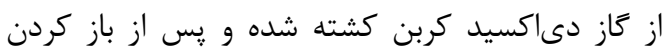

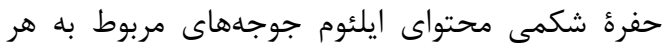

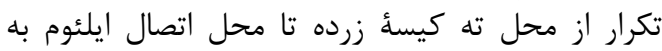

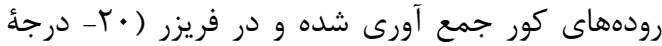

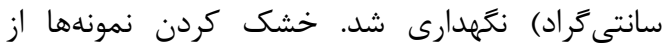

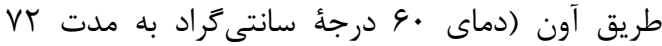

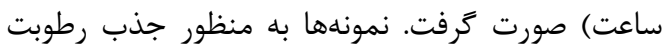

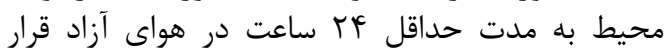

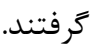

تغذيئ دانهُ كامل به واسطهى عبور آهسته خوراك سبب

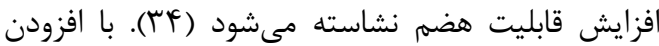

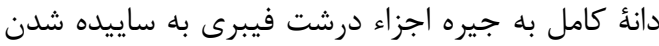

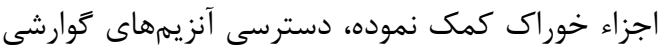

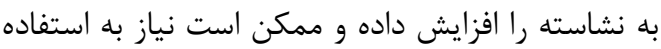

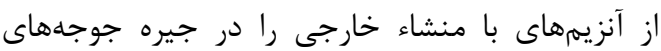

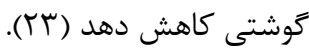

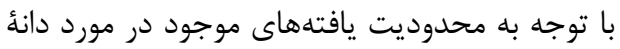

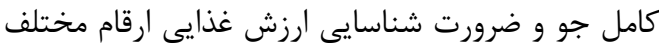

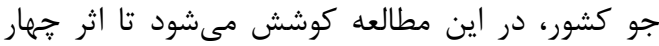

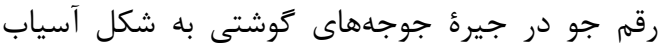

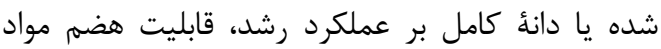

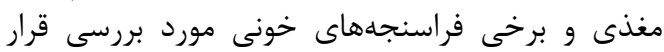
كيرد.

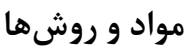

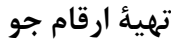

ارقام جو مورد استفاده در اين آزمايش محصول سال

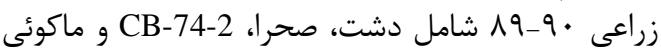
بودند كه از شركت خدمات حمايتى كشاورزى استان اردبيل تهيه شدند.

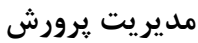

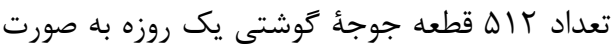

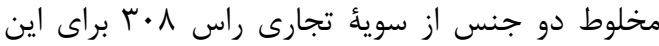

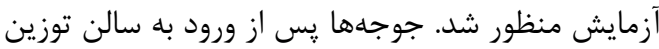

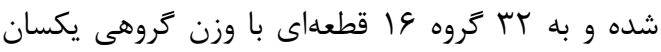

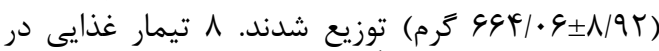

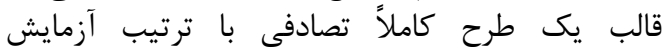

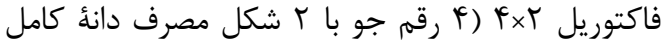

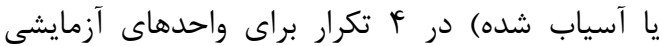

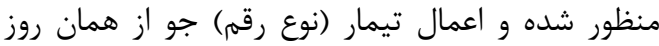

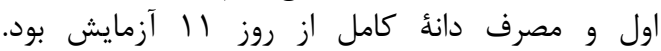

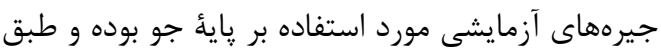

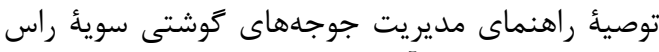

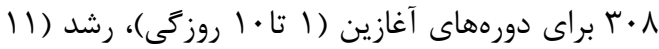



تأثير جهار رقم جو به صورت دانه كامل يا آسياب شده بر عملكرد، قابليت هضم مواد مغذى و فراسنجههاى ليييدى

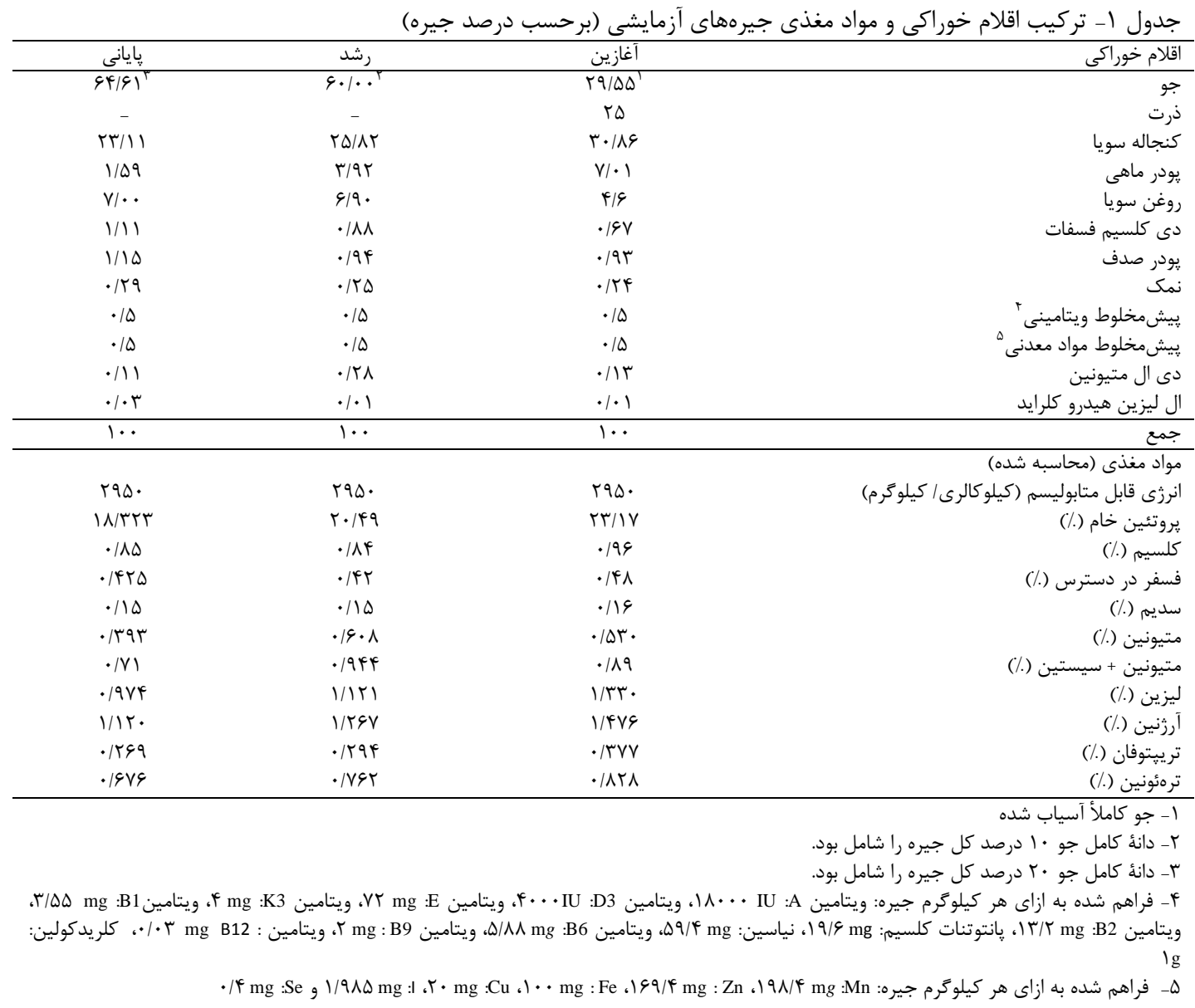

تعيين (DV++ pro مدل ماخت آمريكا Brookfield)

شد.

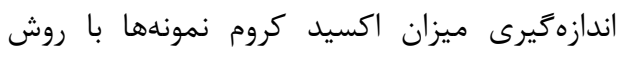

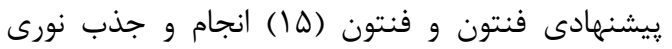

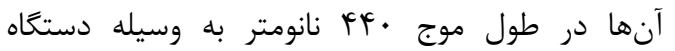

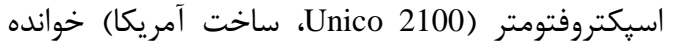
شد. سيس قابليت هضم مواد مغذى و انرزى في با استفاده از فرمول زير تعيين شد (ب) (ب).

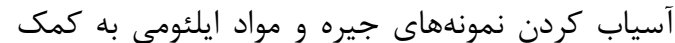

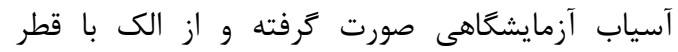

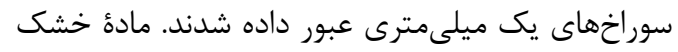

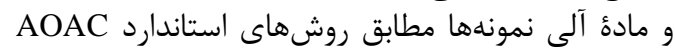

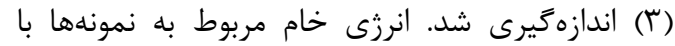

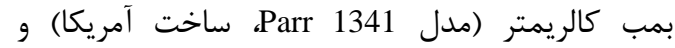

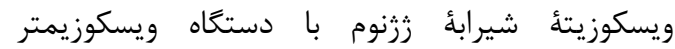

$$
\text { ماده مغذى نمونه ايلئومى(\%) } \times 100
$$

كلسترول كل، كلسترول HDL و ترى كليسريد با روش

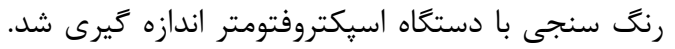

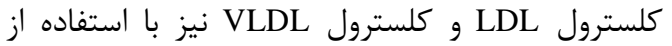
فرمولهاى زير محاسبه شدند (1)

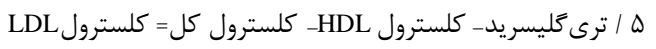

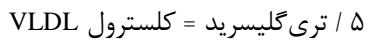

اندازهيرى فراسنجههاى خونى

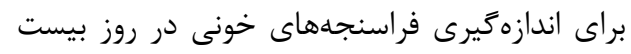

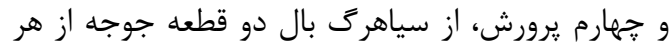

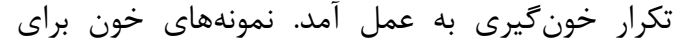
جداسازى سرم با دستخاه سانتريفيوز (VISION VS-15000 CFN II

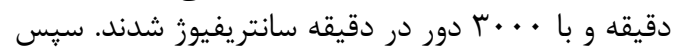

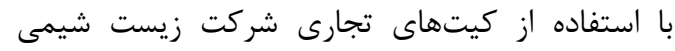


طورى كه جوجههاى تغذيه شده با جيرههاى حاوى دانه

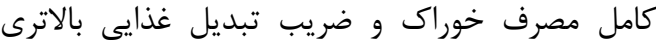

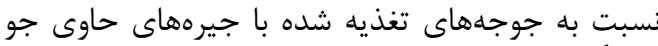

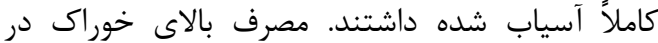

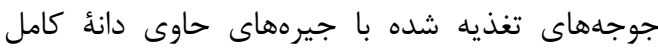

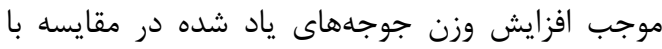

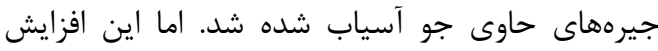

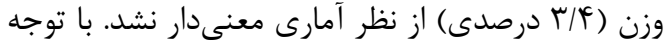

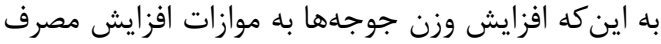

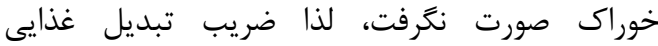

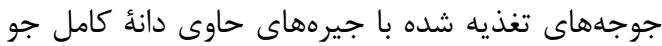

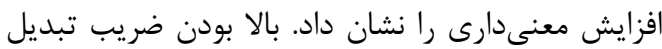

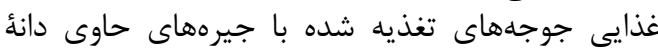

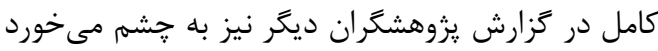

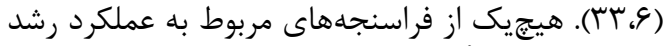

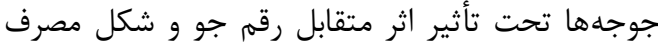

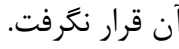

براى تجزيه و تحليل آمارى دادههاى به دست آماري آمده

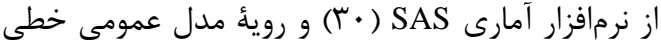
آن استفاده شد. ميانكَين دادهها نيز با استفاده آنداه

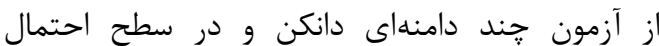
(P< با هم مورد مقايسه قرار كرفتند.

نتايج و بحث

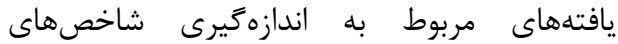

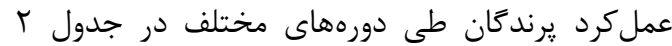

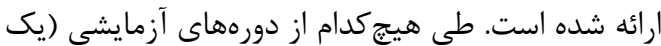

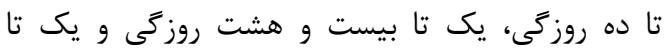

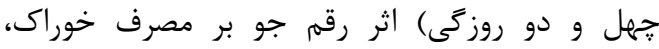

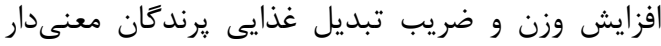

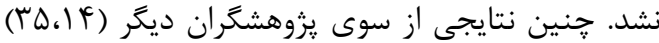

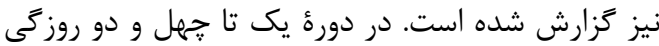

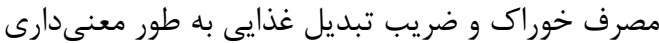

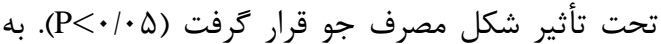

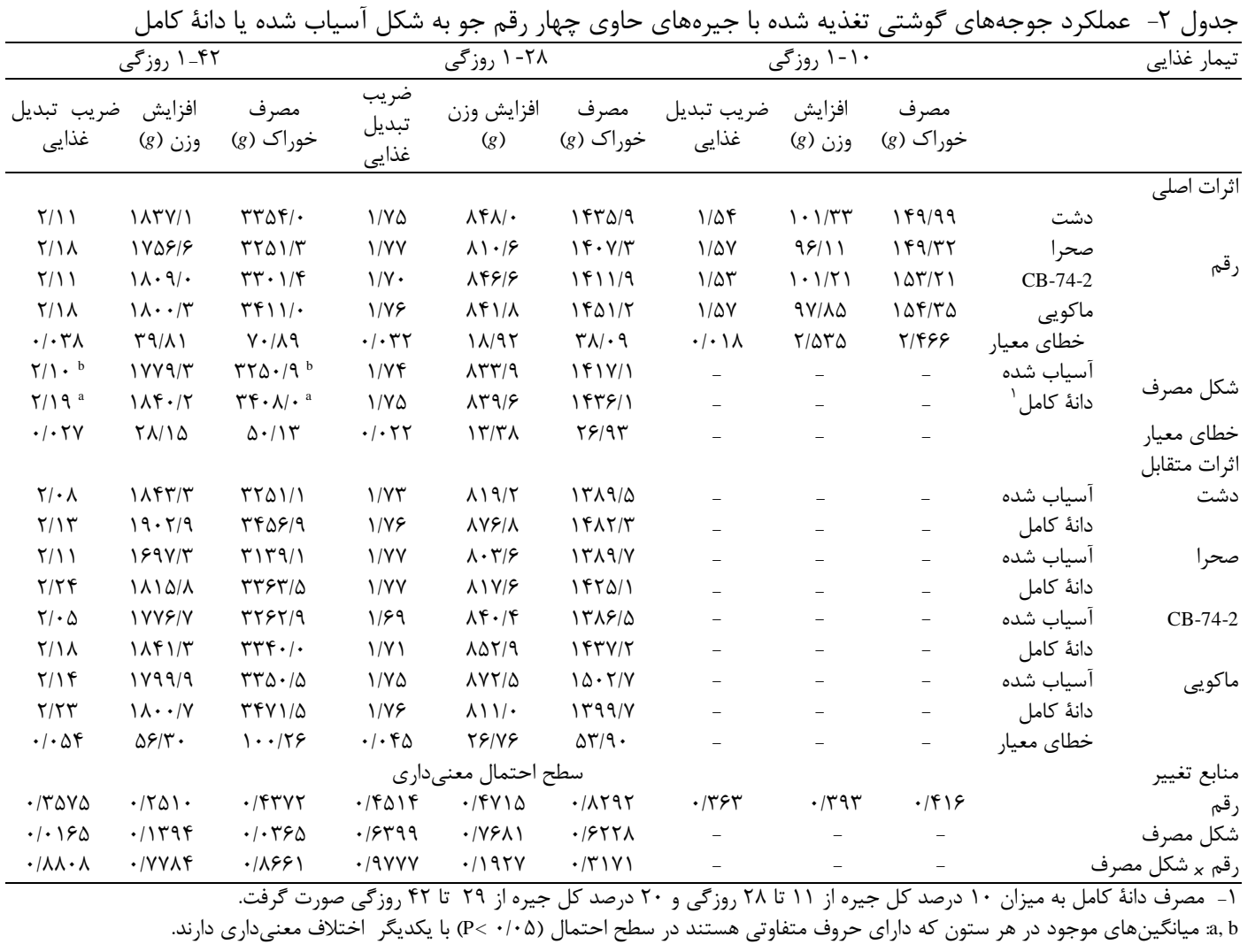

شده است. جوجههاى تغذيه شده با جيرءٔ حاوى رقم

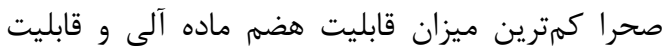

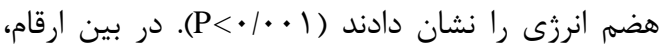

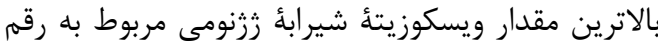

اطلاعات مربوط به قابليت هضم ايلئومى مواد مغذى تغذي

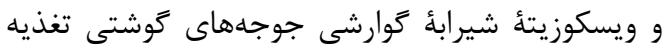

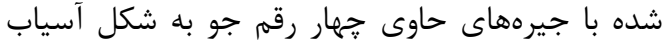

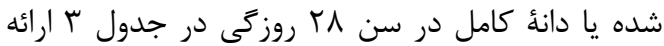


كوشتى تغذيه شده با جيرههاى حاوى جو لخت در اثر اثر

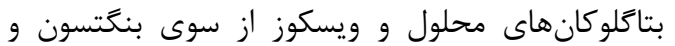

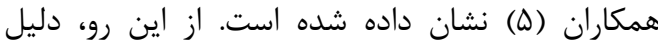

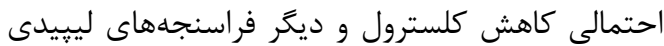

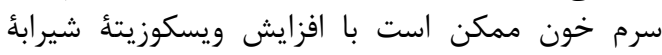

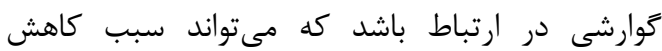

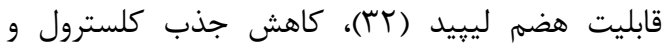

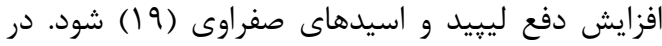

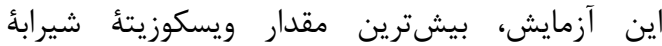

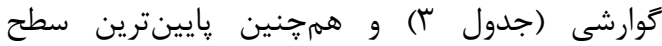

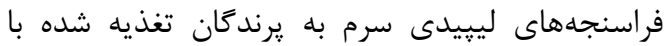

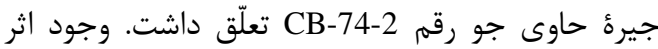

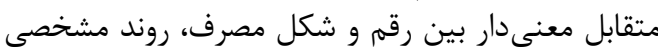

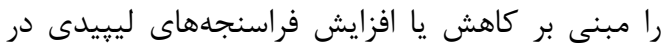

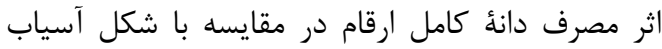

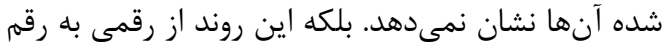

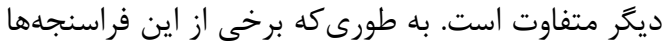

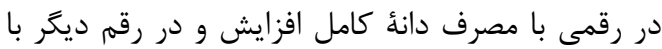

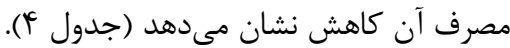

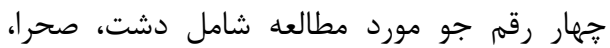

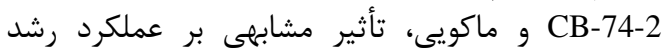

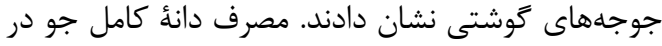

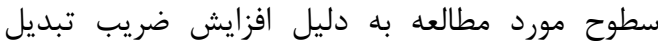

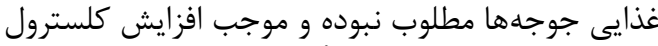

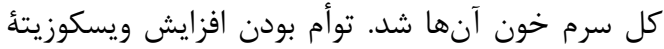

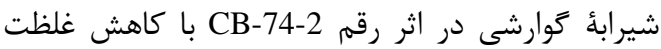

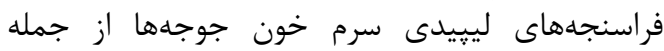

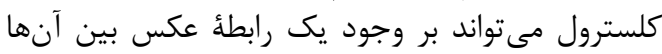

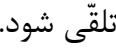

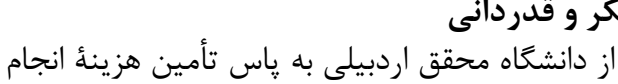

$$
\text { اين يزوهش قدردانى مىشود. }
$$

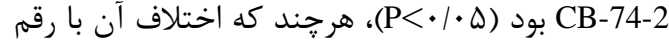

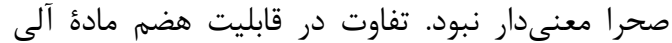

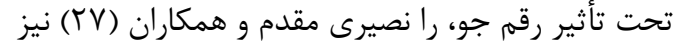

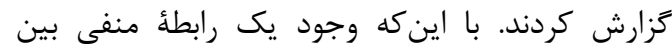

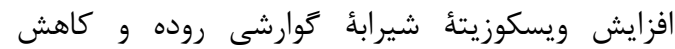

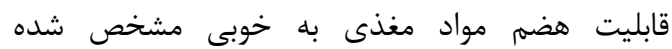

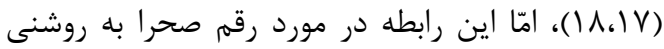

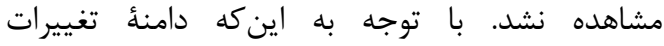

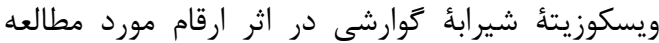

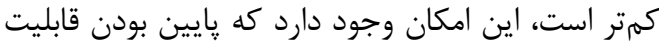

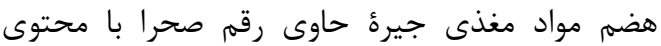

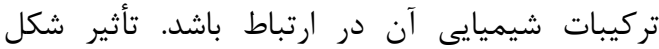

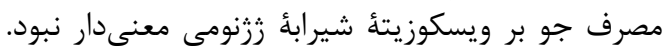

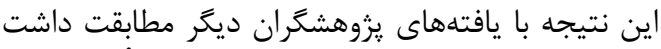

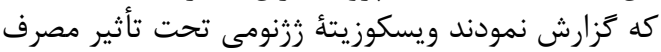

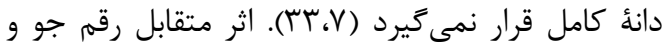

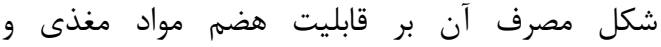

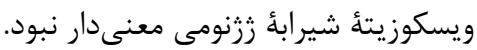

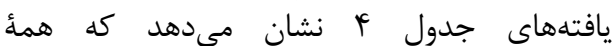

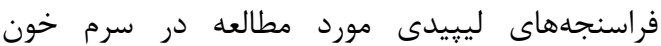

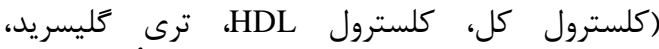

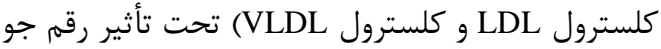

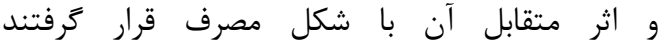

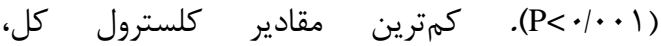
ترى گليسريد، كلسترول LDL و كلسترول

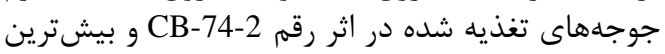

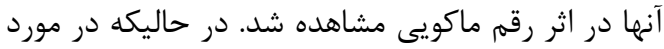

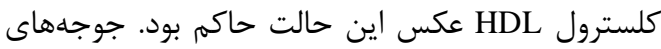

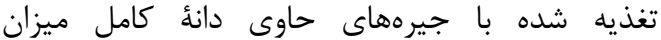

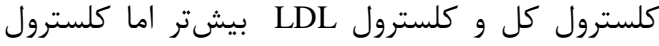

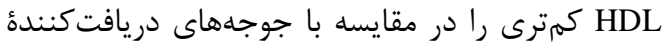

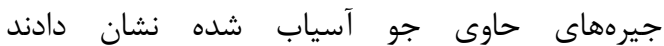
(P</ (P). كاهش ميزان كلسترول خون جوجهاي 


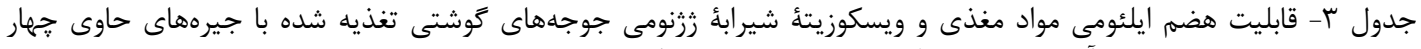

\begin{tabular}{|c|c|c|c|c|c|}
\hline \multirow{2}{*}{ ويسكوزيته } & \multirow[b]{2}{*}{ 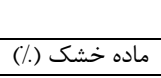 } & \multicolumn{4}{|c|}{ رقم جو به شكل آسياب شده يا دانهُ كامل در سن ^ץ روزگى } \\
\hline & & 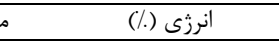 & ماده آلى (.) () ماد & & تي ليمار غذايى \\
\hline$r / \Gamma \Delta^{\mathrm{D}}$ & $4 \% / \Lambda \Delta$ & $99 / \mathrm{Vq}^{\mathrm{a}}$ & $9 N / q \mathrm{r}^{\mathrm{a}}$ & دشت & اثرات اصلى \\
\hline$r / \Delta \gamma^{a b}$ & sT/rם & $\Delta Q / \wedge V^{0}$ & $g r / \Lambda T^{D}$ & صحرا & 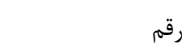 \\
\hline$r / 9 a^{a}$ & $99 / 40$ & $9 D / \Gamma^{\mathrm{a}}$ & $9 N / T \Delta^{\mathrm{a}}$ & CB-74-2 & \\
\hline$r / 1.0$ & $9 \Delta / \Lambda F$ & GN/VDa & $V \cdot \mid \Delta \Lambda^{\mathrm{a}}$ & ماكويى & \\
\hline ./IVG & I/ATV & $\cdot / V \Delta V$ & .948 & خطاى معيار & \\
\hline ५/৭9 & sF/V9 & 9N/9. & s9/9 & آسياب شده & \\
\hline$r / T \Delta$ & $99 / 95$ & 9N/91 & $v 1 / r$. & دانة كامل & 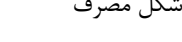 \\
\hline$\cdot / \pi \Delta$. & r/19. & I/DIF & I/ADT & خطاى معيار & \\
\hline & & & & & اثرات متقابل \\
\hline T/T & $9 v / \pi$. & $9 \Delta / 9 \mathrm{~V}$ & 9N/D. & آسياب شده & دشت \\
\hline $\mathrm{r} / \Lambda$ & $9 \pi / \Delta$. & SV/GT & 9N/VY & 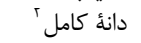 & \\
\hline r/9r & $9 \mathrm{~T} / \mathrm{QV}$ & $91 / .1$ & 94.1 & آسياب شده & صحرا \\
\hline$r / \omega$. & ST/N & $\Delta \Lambda / V Y$ & $91 / 09$ & دانة كامل & \\
\hline$r / \Delta F$ & $9 \mathrm{~V} / 9 \mathrm{~F}$ & 99199 & $v .1 .9$ & آسياب شده & СВ-74-2 \\
\hline f/YG & $94 / 90$ & $9 \pi / v \Delta$ & $99 / 40$ & دانة كامل & \\
\hline r/94 & SF/NG & 9N/9. & 99/9V & آسياب شده & 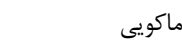 \\
\hline$r / r \Delta$ & $99 / 95$ & 9N/91 & $V \backslash / \Gamma$. & دانة كامل & \\
\hline$\cdot / \pi \Delta$. & r//9. & $1 / \Delta / f$ & 1/AQT & خطاى معيار & \\
\hline & & سطح احتمال معنى دار بودن & & & منابع تغيير \\
\hline .1 .11 & . /K99V &.$\cdots \Delta$ & . $\cdots r$ & آسياب شده & رقم \\
\hline . & . &.$/ 4990$ & . / Tצя & دانه كامل & شكل مصرف \\
\hline - ITATA & - MTAF & $\cdot 19 \cdot v^{k}$ & $\cdot / r \Delta \Delta F$ & & رقمه شكل مصرف \\
\hline
\end{tabular}

جدول f- فراسنجههاى ليبيدى سرم خون جوجههاى كوشتى تغذيه شده با جيرههاى حاوى جهار رقم جو به شكل آسياب شده

\begin{tabular}{|c|c|c|c|c|c|c|}
\hline $\begin{array}{c}\text { VLDL كلسترول(mg/dl) } \\
\text { (mg) }\end{array}$ & $\begin{array}{c}\text { LDL كلسترول/dl) } \\
\text { (mg/d }\end{array}$ & $\begin{array}{c}\text { HDL كلسترول } \\
\text { (mg/dl) }\end{array}$ & كلسترول كل & ترى كليسريد $\begin{array}{c}(m g / d l) \\
\text { (mg }\end{array}$ & & تيمار غذايى \\
\hline & & & & & & اثرات اصلى \\
\hline $11 / \pi 9^{0}$ & $r N / F)^{D}$ & $V 1 / 9 \varphi^{\mathrm{DC}}$ & $|r| /\left.F\right|^{D}$ & $\Delta G / F f^{D}$ & دشت & \\
\hline $11 / \pi \Lambda^{0}$ & $r V / \Delta \AA^{D}$ & $V r / \mu F^{0}$ & $\mid r T / \mathrm{rq}^{D}$ & $\Delta G / K T^{\circ}$ & صحرا & رقم - رقم \\
\hline$N / 4 \varepsilon^{c}$ & $r \mid q Y^{\mathrm{C}}$ & $\Lambda \cdot / 9 q^{a}$ & $111 / \cdot r^{\mathrm{c}}$ & $F T / T^{c}$ & СВ-74-2 & \\
\hline $1 r / \mu r^{a}$ & $r \Delta / \wedge \phi^{\mathrm{a}}$ & $9 N / 09^{\circ}$ & $\mid r \Lambda / r q^{a}$ & $99 / 9 D^{\mathrm{a}}$ & ماكويى & \\
\hline . & $r / \cdot V \wedge$ & 1/זrו & INAFT & 1/ब99 & خطاى معيار & \\
\hline $11 / \pi r$ & $r q / V r^{D}$ & $V \Delta / \Lambda \Delta^{\mathrm{a}}$ & $\| \mid g / 4 \Lambda^{\mathrm{D}}$ & $\Delta S / 9 \Delta$ & آسياب شده & \\
\hline $11 / 19$ & $r Y / \varphi^{a}$ & $V Y / \Delta \varphi^{\circ}$ & $|r \Delta| \cdot \Lambda^{\mathrm{a}}$ & $\Delta \Delta / v \wedge$ & دانة كامل & شكل مصرف \\
\hline . Krs & $1 / 499$ &.$/ 941$ & I/TrT & $1 / / r 1$ & خطاى معيار & \\
\hline & & & & & & اثرات متقابل \\
\hline $\begin{array}{l}1 . / 19^{\circ} \\
1 \% / A \Delta^{\circ}\end{array}$ & $\begin{array}{l}r 9 / 9 Q^{2} \\
r V / \Delta r^{\circ}\end{array}$ & $\begin{array}{l}A F / q Y^{-a} \\
\Delta Q / /\left.\right|^{c}\end{array}$ & $\begin{array}{l}\mid r F / \cdot \Delta^{0} \\
\| 9 / \pi r^{0}\end{array}$ & $\begin{array}{l}Q \cdot / 99^{\circ} \\
91 / 9 Y^{\circ}\end{array}$ & دآسئ كاب شده & دشت \\
\hline $1 Y / 9 \varphi^{\circ}$ & $r \Psi / q Y^{D}$ & $9 r / 99^{\circ}$ & $119 / \Delta V^{0}$ & $G F / N Y^{\circ}$ & آسياب شده & \\
\hline$q / q Y^{c}$ & $\Gamma / / \Delta \Lambda^{c}$ & $\Lambda r / /^{\mathrm{a}}$ & $\mid r \Delta / r^{0}{ }^{0}$ & $r \wedge / Y^{c}{ }^{c}$ & دانٔ كامل & صحرا \\
\hline $9 / 9 .^{\circ}$ & $\mid F / F \psi^{\mathrm{a}}$ & $\Lambda T / V Q^{a}$ & $1 \cdot r / \Lambda \cdot c^{c}$ & $r 1 / 9 \Delta^{a}$ & آسياب شده & \\
\hline $1 \cdot / \Delta r^{c}$ & $r N / Y \Lambda^{C}$ & $v 9 / / r^{a}$ & $\| N / F \Lambda^{D}$ & $\Delta r / q V^{c}$ & دانة كامل & CB-74-2 \\
\hline $10 / v^{a}$ & $r \cdot \pi r^{c}$ & $V \psi / / \Lambda^{0}$ & $119 / 49^{0}$ & $\vee N / 9 \Delta^{\mathrm{a}}$ & آسياب شده & \\
\hline $\mid T / \cdot \Lambda^{0}$ & $91 / \cdot 1^{\mathrm{a}}$ & $9 r / \Lambda \cdot{ }^{c}$ & $\mid r \mu / r q^{a}$ & $4 \cdot / \pi \Lambda^{0}$ & دانةُ كامل & ماكويى \\
\hline . Far & ५/१५q & I/ANr & T/AGF & T/YqI & خطاى معيار & \\
\hline & لون ودن & سطح احتمال معنى & & & & منابع تغيير \\
\hline$<\cdot|\cdots|$ & $<\cdot|\cdots|$ & $<\cdot|\cdots|$ & $<\cdot|\cdots|$, & $<\cdot|\cdots|$ & & رقم \\
\hline.$/ \Delta 94 V$ & $<\cdot|\cdots|$ &...$r v$ & $<\cdot|\cdots|$ & .1091. & & شكل مصرف \\
\hline$<\cdot|\cdots|$ & $<\cdot|\cdots|$, &.$\ldots p$ &.$/ \ldots r$ & $<\cdot|\cdots|$ & & رقهم شكل مصرة \\
\hline
\end{tabular}



of Jihad-e-Agriculture, $123 \mathrm{pp}$ (In Persian).

2. Abdulilah, H.M. 1995. Barley varieties, enzyme supplementation and broiler performance. Applied Poultry Research, 4: 230-234.

3. AOAC. 2000. Official Methods of Analysis, 17th edn. Association of Official Analytical Chemists, Arlinton, VA, USA

4. Bedford, M.R. 1995. Mechanism of action and potential environmental benefits from the use of feed enzymes. Animal Feed science and Technology, 53: 145-155.

5. Bengtsson, S., H. Aman, C.W. Graham and R.K. Newman. 1990. Chemical studies on mixed- linked $\beta$-glucans in hull-less barley cultivars given different hypocholestrolaemic responces in chickens. Journal of the Science of Food and Agriculture, 52: 435-445.

6. Bennett, C.D., H.L. Classen and C. Riddell. 2002. Feeding broiler chickens wheat and Barley diets containing whole, ground and pelleted grain. Poultry Science, 81: 995-1003.

7. Bennett, C.D., H.L. Classen, K. Schwean and C. Riddell. 2002. Influence of Whole Barley and Grit on Live Performance and Health of Turkey Toms. Poultry Science, 81:1850-1855.

8. Bird, F.N. 1971. Distribution of trypsin and $\beta$-Amylase activities in the duodenum of the domestic fowl. British Poultry Science, 12: 373-378.

9. Brand, T.S., C.W. Cruywagen, D.A. Brandt, M. Viljoen and W.W. Burger. 2003. Variation in the chemical composition, physical characteristics and energy values of cereal grains produced in the Western Cape area of South Africa. South African Journal of Animal Science, 33: 117-126.

10. Burnet, G.S. 1966. Studies of viscosity as the probable factor in volved in the improvement of certain barleys for chickens by enzym supplementation. British Poultry Science, 7: 55-75.

11. Cummings, R.B. 1994. Opportunities for whole grain feeding. Proceedings of the 9th European Poultry Conference, Glasgow, UK219-222 pp.

12. Duke, G.E. 1982. Gastrointestinal motility and its regulation. Poultry Science, 61: 1245-1256.

13. Ehsani, M., V. Baratian and M. Torki. 2011. Performance of broilers fed barley-based diets supplemented by two sourses of commercial probiotics. World Applied Science Journal, 14 (Special Issue of Food and Environment): 09-14.

14. Farran, M.T., G. Barbour, N.N. Usayran, A.H. Darwish, H.H. Machlab, M. Hruby and V.M. Ashkarian. 2010. Performance and carcass quality of broiler chickensfed a corn-soybean meal diet containing graded barley levels without enzyme. Journal of Poultry Science, 47: 34-40.

15. Fenton, T.W. and M. Fenton. 1979. An improved procedure for the determination of chromic oxid in feed and feces. Canadian Journal of Animal Science, 59: 631-634.

16. Friedwald, W.T., R.I. Levy and D.S. Fredrickson. 1972. Estimation of concentration of Low-density lipoprotein in plasma without use of the ultra-centrifuge. Clinical Chemistry, 18: 449-502.

17. Friesen, O.D., W. Guenter, R.R. Marquardt and B.A. Rotter. 1992. The effect of enzyme supplementation on the apparent metabolizable energy and nutrient digestibilitiy of wheat, barley, oat, and rye for broiler chick. Poultry Science, 71: 1710-1721.

18. Fuente, J.M., P.P. De Ayala, A. Flores and M.J. Villamide. 1998. Effect of storage time and dietary enzymes on the metabolizable energy and digesta viscosity of barley-based diets for poultry. Poultry Science, 77: 90-97.

19. Gallaher, C.M., J. Munion, J.R. Hesslink, J. Wise and D.D.R. Gallaher. 2000. Cholestrol reduction by glucomanan and chitosan is mediated by changes in cholestrol absorption and bile acd and fat excretion in rats. Journal of Nutrition, 130: 2753-2759.

20. Gheisari, A.A., A.H. Pourabadeh, J. Pourreza, M. Mahlougi and R. Bahadoran. 2007. Determination of chemical composition and evaluation of apparent and true metabolisable energy of different barley cultivars in broiler chicks. Journal of Sciences and Technology of Agriculture and Natural Resources, Water and Soil science, 11: 405-415 (In Persian).

21. Janmohammadi, H., A. Taghizadeh and N. Pirany. 2009. Chemical composition and metabolizale energy content of some barley varieties of east azarbyjan using adult Leghorn roosters. Journal of agricultural science (University of Tabriz), 19: 105-115 (In Persian).

22. Jeroch, H. and S. Danicke. 1995. Barley in poultry feeding: a review. World's Poultry Science Journal, 51: 271-291.

23. Jones, G.P.D. and R.D. Taylor. 2001. The incorporation of whole grain into pelleted broiler chicken diets: production and pHysiological responses. British Poultry Science, 42: 477-483.

24. Kato, T., A. Sasali and G. Taleda. 1995. Genetic variation of $\beta$-glucan contents and $\beta$-glucanase activities in barley and their relationships to malting quality. Breeding Science, 45: 476-477.

25. Mc Intosh, J.I., S.J. Slinger, I.R. Sibbald and G.C. Ashton. 1962. Factors affecting the metabolizable energy content of poultry feed. 7 . The effects of grinding, pelleting and grit feeding on the availability of the energy of wheat, corn, oats and barley. 8. A study on the effects of dietary balance. Poultry Science, 41: 445-456.

26. Narasimhalu, P., D. Kong, T.D. Choo, T. Ferguson, M.C. Therrien, K.M. Ho, K.W. May and P. Jui. 1995. Effects of environment and cultivar on total mixed-linkage $\beta$-glucan content in eastern and western Canadian barleys (Hordeum vulgare L.). Canadian Journal of Plant Science, 75: 371-376.

27. Nasiri moghadam, H., M. Danesh mesgaran and M.D. Shakouri. 2006. Nutritive value comparison of different barley cultivars with and without enzyme supplementation in broiler nutrition. Agricultural sciences and technology, 20: 57-68 (In Persian).

28. National Research Council. 1994. Nutrient Requirements for Poultry. National Academy Press, Washington DC. 
29. Pourreza, J., G. Sadeghi and M. Mehri. 2005. Scott's Nutrition of the Chicken, Arkan, Isfahan, Iran, 668 pp (In Persian).

30. Saha, D.C. and R.L. Gilbreat. 1993. A modified chromic oxide indicator ratio technique for accurate determination of nutrient digestibility. Canadian Journal of Animal Science, 73: 1001-1004.

31. SAS Institute. 2002. SAS/STAT User's guide: Statistics. Version 9.1. $4^{\text {th }}$ ed. SAS Inst. Inc., Cary, NC.

32. Smits, C.H.M., A. Veldman, H.J. verkade and A.C. Beynen. 1998. The inhibitory effect of carboxymethyle cellulose with high viscosity on lipid absorptin in broiler chickens coincides with reduced bile salt concentration and raised microbial numbers in the small intestine. Poultry Science, 77: $1534-1539$

33. Svihus, B., O. Herstad, C.W. Newman and R.K. Newman. 1997. Comparison of performance and intestinal characteristics of broiler chickens fed on diets containing whole, rolled or ground barley. British Poultry Science, 38: 524-529.

34. Svihus, B. and H. Hetland. 2001. Ileal starch digestibility in growing broiler chickens fed on a wheat based diet is improved by mash feeding, dilution with cellulose or whole wheat inclusion. British Poultry Science, 42: 633-637.

35. Torki, M. 2006. Effects of Barley Cultivars and Probiotic Supplementation on Growth Performance of Broiler Chicks Fed on Barley-based Diets, XII European Poultry Conference, Sep 10-14, Verona, Italy.

36. Yoon, S.H., P.T. Berglund and C.E. Fastnaught. 1995. Evaluation of selected barley cultivars and their Fractions for $\beta$-glucan enrichment and viscosity. Cereal Chemistry, 72: 187-190.

37. Zhang, G., J. Chen, J. Wang and S. Ding. 2001. Cultivar and Environmental Effects on $(1 \rightarrow 3,1 \rightarrow 4)-$ $\beta$-D-glucan and Protein Content in Malting Barley. Journal of Cereal Science, 34: 295-301. 


\title{
The Effect of Four Barley Cultivars in Whole and Ground Forms on Performance, Nutrients Digestibility and Blood Lipid Parameters of Broiler Chickens
}

\begin{tabular}{c} 
Mohammad Malekzadeh $^{1}$ and Mir Daryoush Shakouri \\
\hline \hline 1- Ph.D. Student, University of Mohaghegh Ardabili \\
2- Assistant Professor, University of Mohaghegh Ardabili \\
(Corresponding author: mdshakouri@ uma.ac.ir) \\
Received: December 5, 2013 $\quad$ Accepted: April 23, 2014 \\
\hline \hline
\end{tabular}

\begin{abstract}
This experiment was conducted to evaluate the effect of barley cultivars and their feeding form on performance, jejunal digesta viscosity, nutrients digestibility and blood parameters of broiler chickens. A total of 512 day-old chicks (Ross 308), as mixed sex, were assigned to experimental treatments using a completely randomized design with factorial arrangement $(4 \times 2)$ for a period of 42 days. Barley cultivars were Dasht, Sahra, CB-74-2 and Makoei and feeding forms were in whole and ground as well. According to the results, barley cultivar had no significant effect on broilers performance and feeding whole grain increased feed intake and feed conversion ratio of the birds $(\mathrm{P}<0.05)$. On day 28 , the highest amount of jejunal digesta viscosity $(\mathrm{P}<0.05)$ and the lowest digestibility of organic matter and energy $(\mathrm{P}<0.001)$ were observed by CB-74-2 and Sahra cultivars, respectively. The birds receiving Makoei cultivar had the highest level of triglyceride, total cholesterol, LDL-cholesterol and VLDL-cholesterol in their sera at 24 days of age $(\mathrm{P}<0.0001)$. Ingestion of whole grain increased total and LDLcholesterol and decreased HDL-cholesterol levels of the sera $(\mathrm{P}<0.01)$. Based on the results, while the barley cultivars showed different effects on some studied parameters, they had similar influence on broiler growth performance and their whole feeding form failed to improve the birds' performance.
\end{abstract}

Keywords: Barley cultivar, Broiler chickens, Jejunal digesta viscosity, Nutrient digestibility, Performance, Whole grain 